\title{
Rovibrational Interaction and Vibrational Constants of the Symmetric Top Molecule ${ }^{14} \mathrm{NF}_{3}$
}

\author{
Hamid Najib \\ Laboratoire de Physique de la Matière Condensée, Équipe de Spectrométrie Physique, Département de Physique, \\ Université Ibn Tofaïl, Faculté des Sciences, BP 133, 14000 Kénitra, Morocco
}

Correspondence should be addressed to Hamid Najib; najib195@gmail.com

Received 8 April 2013; Accepted 8 May 2013

Academic Editors: A. F. Borghesani and A. Kochur

Copyright (C) 2013 Hamid Najib. This is an open access article distributed under the Creative Commons Attribution License, which permits unrestricted use, distribution, and reproduction in any medium, provided the original work is properly cited.

Several accurate experimental values of the $\alpha^{C}$ and $\alpha^{B}$ rotation-vibration interaction parameters and $\omega_{i}, x_{i j}$, and $g_{i j}$ vibrational constants have been extracted from the most recent high-resolution Fourier transform infrared, millimeter wave, and centimeter wave investigations in the spectra of the oblate symmetric top molecule ${ }^{14} \mathrm{NF}_{3}$. The band-centres used are those of the four fundamental, the overtones, the combination, and hot bands identified in the region between $400 \mathrm{~cm}^{-1}$ and $2000 \mathrm{~cm}^{-1}$. Comparison of our constants with the ones measured previously, by infrared spectroscopy at low resolution, reveals orders of magnitude higher accuracy of the new values. The agreement between our values and those determined by ab initio calculations employing the TZ2Pf basis is excellent.

\section{Introduction}

Nitrogen trifluoride $\mathrm{NF}_{3}$ is a pyramidal symmetric top molecule belonging to $\mathrm{C}_{3 \mathrm{v}}$ point group. It has four vibrational normal modes:

(i) two totally symmetric ( $A_{1}$ type): $v_{1}$ and $v_{2}$;

(ii) two doubly degenerate ( $E$ type): $\nu_{3}$ and $\nu_{4}$.

Today, $\mathrm{NF}_{3}$ is employed in the cleaning of the PECVD chambers in the high volume production of liquid crystal displays and silicon-based thin film solar cells. It has been considered as an environmentally preferable substitute for sulphur hexafluoride or perfluorocarbons such as hexafluoroethane [1]. But, recent publications [2-7] reported that $\mathrm{NF}_{3}$ is 17,000 times more potent a greenhouse gas than $\mathrm{CO}_{2}$ and recommended to include it in the basket of gases controlled by Kyoto protocol and to monitor its environmental levels.

Nitrogen trifluoride is also used in hydrogen fluoride and deuterium fluoride lasers, which are types of chemical lasers.

In spite of these interesting properties and important applications, the spectra of this molecule have not yet been much studied in the past, because of difficulties to obtain high quality spectra and to interpret its perturbed bands. Many of these difficulties have now been overcome through the advent of high-resolution spectrometers and the development of computers.

This is within the framework of this research orientation and the Moroccan/European collaboration that several bands of $\mathrm{NF}_{3}$ gas were recently analysed [8-14]. We used independent and complementary methods, Fourier transform infrared (FTIR), millimetre wave (MMW), and centimetre wave (CMW) spectroscopies. All studied IR spectra were recorded at Wuppertal (Germany) with the Bruker $120 \mathrm{HR}$ interferometer, except for the region around $1032 \mathrm{~cm}^{-1}$, which was recorded at the University of Giessen (Germany). The rotational spectra were measured in Lille (France) with a computer-controlled MMW spectrometer. The measurements in the CMW range were performed at Kiel (Germany) by means of wave-guide microwave Fourier transform spectroscopy.

The subject of this work is to extract accurate experimental rotation-vibration interaction parameters and anharmonicity constants of the potential function of ${ }^{14} \mathrm{NF}_{3}$ from 
our recent studies by high-resolution spectroscopy and the latest results available in the literature.

\section{Relationships Linking Vibrational Constants}

More recently, we have established the following relationships linking band-centres, harmonic wavenumbers, and anharmonicity constants of a symmetric top molecule [15].

\subsection{Fundamental Bands $v_{i}=1$}

$$
\left(v_{i}^{\ell_{i}}\right)=\omega_{i}^{0}+x_{i i}+g_{i i} \ell_{i}
$$

$\omega_{i}$ is the normal wavenumber of the $i$ th mode; $v_{i}$ is its vibrational quantum number; $i$ refers to a totally symmetric or to a degenerate vibration; $x_{i i}$ and $g_{i i}$ are the anharmonicity constants; and $\ell_{i}$ is the vibrational angular momentum: $\left|\ell_{i}\right|=$ $v_{i}, v_{i}-2, \ldots, 1$, or 0 only exists for a doubly degenerate vibration.

\subsection{Harmonic Bands $v_{i}=n$ (Integer $\left.>1\right)$}

$$
\left(n v_{i}^{\ell_{i}}\right)=n\left(v_{i}^{ \pm 1}\right)^{0}+\left(n^{2}-n\right) x_{i i}+\left(\ell_{i}^{2}-n\right) g_{i i}
$$

2.3. Combination Bands $v_{i}=n$ and $v_{i^{\prime}}=m$ (Integer), $i \neq i^{\prime}$

$$
\begin{aligned}
\left(n v_{i}^{\ell_{i}}+m v_{i^{\prime}}^{\ell_{i^{\prime}}}\right)^{0}= & \left(n v_{i}^{\ell_{i}}\right)^{0}+\left(m v_{i^{\prime}}^{\ell_{i^{\prime}}}\right)^{0} \\
& +n m x_{i i^{\prime}}+g_{i i^{\prime}} \ell_{i} \ell_{i^{\prime}} .
\end{aligned}
$$

For the oblate symmetric top $\mathrm{NF}_{3}$, we count $4 \omega_{i}^{0}, 10 x_{i j}$, and $3 g_{i j} ; i \leq j$.

\section{The Ground State Constants of ${ }^{14} \mathbf{N F}_{3}$}

The experimental ground state (GS) axial rotational parameters for the symmetric top molecule ${ }^{14} \mathrm{NF}_{3}$ employed in the present study and reported in Table 1 come from different sources.

For the $C_{0} K$-dependent constant, we used in our highresolution studies the following values:

(i) $C_{0}=0.1949980$ (10): a preliminary value obtained in the course of our work on the $2 v_{4}$ overtone band of ${ }^{14} \mathrm{NF}_{3}$,

(ii) $C_{0}=0.19499250$ (44): the definitive value adopted in the study of the $v_{4}=2$ state of ${ }^{14} \mathrm{NF}_{3}$. In the two cases, we used the "loop method" as described in [16].

For the $B_{0} J$-dependent constant, the values adopted were determined by Höhe et al. [17], Breidung et al. [18], and Cazzoli and Puzzarini [19].
TABLE 1: Ground state constants $\left(\mathrm{cm}^{-1}\right)$ of ${ }^{14} \mathrm{NF}_{3}$.

\begin{tabular}{lccc}
\hline Parameter & Value $[12,17]$ & Value $[11,18]$ & Value $[11,19]$ \\
\hline$C_{0}$ & $0.1949980(10)$ & $0.19499250(44)$ & $0.19499250(44)$ \\
$B_{0}$ & $0.3562828965(40)$ & $0.3562827950(15)$ & $0.3562828891(21)$ \\
\hline
\end{tabular}

\section{The Rovibrational Bands of ${ }^{14} \mathrm{NF}_{3}$ Observed below $2000 \mathrm{~cm}^{-1}$}

4.1. The $v_{1}$ Fundamental Band [17]. The $v_{1}=1$ excited state of ${ }^{14} \mathrm{NF}_{3}$ was investigated by FTIR spectroscopy and with saturation technique using $\mathrm{CO}_{2}$ side bands as a tunable infrared source. This level was considered unperturbed, and the fit gave the band-centre: $\left(\nu_{1}\right)^{0}=1032.00123750(47) \mathrm{cm}^{-1}$. For the excited rotational constants, the values were determined relative to the GS.

4.2. The $v_{4}$ Fundamental Band [14]. The lowest fundamental band $v_{4}$ of the nitrogen trifluoride ${ }^{14} \mathrm{NF}_{3}$ was studied by high-resolution FTIR, MMW, and CMW spectroscopies near $493 \mathrm{~cm}^{-1}$. The analysis of the $v_{4}=1$ state included $\ell(2,2), \ell(2,-1), \ell(2,-4)$, and $k(0,6)$ intravibrational interactions and gave the band-centre: $\left(v_{4}\right)^{0}=493.4227759$ (89) $\mathrm{cm}^{-1}$.

4.3. The $v_{2}$ Fundamental, $2 v_{2}-v_{2}$ Hot, $2 v_{2}$ Overtone, and $v_{2}+$ $v_{4}$ Combination Bands [13]. They were analysed by FTIR and MMW spectroscopies. The corresponding excited states were treated as isolated levels, and the following band-centres were determined:

$$
\begin{gathered}
\left(v_{2}\right)^{0}=647.1340617(73) \mathrm{cm}^{-1} \\
\left(2 v_{2}-v_{2}\right)^{0}=645.121943(14) \mathrm{cm}^{-1} \\
\left(v_{2}+v_{4}\right)^{0}=1138.276629(10) \mathrm{cm}^{-1}
\end{gathered}
$$

Because of the low intensity of the $2 v_{2}$ overtone, the $v_{2}=2$ state constants were determined from the $2 v_{2}-v_{2}$ hot and $v_{2}$ fundamental bands.

We point out that any explanation was offered for a severe intensity perturbation observed in the spectra of $v_{2}$ and $2 v_{2}$ bands (see [13]).

4.4. The $v_{3}$ Fundamental Band [12]. The perpendicular band $v_{3}$ of the symmetric top ${ }^{14} \mathrm{NF}_{3}$ was analysed by highresolution FTIR and MMW spectroscopies. The $v_{3}=1$ excited state was considered vibrationally isolated, but several intravibrational interactions were included in the final fit. The band-centre obtained is $\left(v_{3}\right)^{0}=907.5413300(72) \mathrm{cm}^{-1}$.

4.5. The $2 v_{4}$ Overtone Band [11]. The $v_{4}=2$ excited state of the oblate molecule ${ }^{14} \mathrm{NF}_{3}$, lying near $985 \mathrm{~cm}^{-1}$, was successfully studied by high-resolution FTIR and MMW spectroscopies. Assumed to be isolated, the treatment of this level gave the 
TABLE 2: Band-centres and rovibrational interaction constants of ${ }^{14} \mathrm{NF}_{3}$ molecule (in $\mathrm{cm}^{-1}$ ).

\begin{tabular}{|c|c|c|c|c|c|}
\hline $\begin{array}{l}\text { Band or } \\
\text { component }\end{array}$ & $\begin{array}{c}\text { Experimental } \\
\text { band-centre, this } \\
\text { work }\end{array}$ & $\begin{array}{l}\text { Experimental } \alpha^{C} \times 10^{3} \text {, } \\
\text { this work }\end{array}$ & $A b$ initio $\alpha^{B} \times 10^{3},[18]$ & $\begin{array}{l}\text { Experimental } \alpha^{B} \times 10^{3}, \\
\text { this work }\end{array}$ & $A b$ initio $\alpha^{B} \times 10^{3},[18]$ \\
\hline$v_{1}$ & $1032.00123750(47)$ & $0.593526(40)$ & 0.643 & $-1.4496825(43)$ & -1.227 \\
\hline$v_{2}$ & $647.1340617(73)$ & $0.4346(10)$ & 0.404 & $1.289844(18)$ & 1.300 \\
\hline$v_{3}$ & $907.5413300(72)$ & $0.7374(11)$ & 0.857 & $2.628451(22)$ & 2.758 \\
\hline$v_{4}$ & $493.4227759(89)$ & $0.5566(10)$ & 0.544 & $0.150021(23)$ & 0.164 \\
\hline $2 v_{2}-v_{2}$ & $645.121943(14)$ & $0.8777(10)$ & - & $2.592650(37)$ & - \\
\hline $2 v_{2}$ & $1292.25004(21)^{\mathrm{a}}$ & $0.8777(10)$ & - & $2.592650(37)$ & - \\
\hline$v_{2}+v_{4}$ & $1138.276629(10)$ & $0.9922(10)$ & - & $1.447060(43)$ & - \\
\hline $2 v_{4}^{0}$ & $983.701767(34)$ & $1.10135(63)$ & - & $0.192090(23)$ & - \\
\hline $2 v_{4}^{ \pm 2}$ & $986.622364(18)$ & $1.10101(56)$ & - & $0.302179(50)$ & - \\
\hline $2 v_{3}^{0}$ & $1803.130213(57)$ & $1.47497(80)$ & - & $4.84308(22)$ & - \\
\hline $2 v_{3}^{ \pm 2}$ & $1810.423993(21)$ & $1.49747(50)$ & - & $5.24715(12)$ & - \\
\hline$v_{1}+v_{3}$ & $1931.577516(19)$ & $1.30958(56)$ & - & $1.460127(78)$ & - \\
\hline$v_{1}+v_{4}$ & $1523.040783(34)$ & $1.37339(84)$ & - & $-1.13109(44)$ & - \\
\hline$v_{2}+v_{3}$ & $1546.30^{\mathrm{b}}$ & - & - & - & - \\
\hline
\end{tabular}

Numbers in parentheses represent one standard deviation in units of the last digit quoted.

${ }^{\mathrm{a}}$ Value extracted from the values of $\left(v_{2}\right)^{0}$ and $x_{22}$ (Tables 2 and 3). ${ }^{\mathrm{b}}$ Value estimated from our FTIR spectrum which is in course of study.

following band-centres for the parallel and perpendicular components:

$$
\begin{aligned}
& \left(2 v_{4}^{0}\right)^{0}=983.701767(34) \mathrm{cm}^{-1} \\
& \left(2 v_{4}^{ \pm 2}\right)^{0}=986.622364(18) \mathrm{cm}^{-1}
\end{aligned}
$$

4.6. The $2 v_{3}$ Overtone and $v_{1}+v_{3}$ Combination Bands [10]. In the analysis of these bands, we used spectra recorded by Fourier transform spectrometer. The same models applied to the $v_{4}=2$ and $v_{3}=1$ excited states were applied to treat the $2 v_{3}$ and $v_{1}+v_{3}$ bands. We obtained the following results:

$$
\begin{gathered}
\left(2 v_{3}^{0}\right)^{0}=1803.130213(57) \mathrm{cm}^{-1}, \\
\left(2 v_{3}^{ \pm 2}\right)^{0}=1810.423993(21) \mathrm{cm}^{-1}, \\
\left(\nu_{1}+v_{3}\right)^{0}=1931.577516(19) \mathrm{cm}^{-1} .
\end{gathered}
$$

4.7. The $v_{1}+v_{4}$ Combination Band [8, 9]. The $v_{1}+v_{4}$ perpendicular band of ${ }^{14} \mathrm{NF}_{3}$ was studied by high-resolution infrared spectroscopy. It was treated by a model taking into account $\ell$ - and $k$-type intervibrational interactions. The band-centre obtained in the $D$-reduction of the rovibrational Hamiltonian is $\left(v_{1}+v_{4}\right)^{0}=1523.040783(34) \mathrm{cm}^{-1}$.

\section{Results and Discussion}

In Table 2, we gather the recent measurements, at highresolution, of the centres of the all studied bands between $400 \mathrm{~cm}^{-1}$ and $2000 \mathrm{~cm}^{-1}$ and the corresponding experimental rotation-vibration interaction constants: $\alpha^{C}=C_{0}-C^{\prime}$ and
$\alpha^{B}=B_{0}-B^{\prime}$ and $C^{\prime}$ and $B^{\prime}$ being the constants of the upper level.

The values of the rovibrational interaction constants were determined by ab initio calculations (Table 2) for only the fundamental bands. The agreement with our values is excellent.

Equation (2) leads to $x_{24}=\left(v_{2}+v_{4}\right)^{0}-\left(v_{2}\right)^{0}-\left(v_{4}\right)^{0}=$ $-2.280208(11) \mathrm{cm}^{-1}$.

For the $2 v_{4}$ harmonic band, we obtain the system of equations:

$$
\begin{aligned}
& \left(2 v_{4}^{0}\right)^{0}=2\left(v_{4}^{ \pm 1}\right)^{0}+2 x_{44}-2 g_{44}, \\
& \left(2 v_{4}^{ \pm 2}\right)^{0}=2\left(v_{4}^{ \pm 1}\right)^{0}+2 x_{44}+2 g_{44} .
\end{aligned}
$$

We derive $x_{44}=-0.841743(22) \mathrm{cm}^{-1}$ and $g_{44}=0.730149(21)$ $\mathrm{cm}^{-1}$.

As for $2 v_{4}$, we deduce $x_{33}=-4.15278(18) \mathrm{cm}^{-1}$ and $g_{33}=$ $1.82344(18) \mathrm{cm}^{-1}$.

Using the band-centres of $v_{1}$ and $v_{4}$, we obtain $x_{14}=$ $-2.383248(49) \mathrm{cm}^{-1}$. We can also derive, from the bandcentres of $v_{1}$ and $v_{3}$, the anharmonic constant: $x_{13}=-7.965051$ (26) $\mathrm{cm}^{-1}$.

All experimental anharmonicity constants determined in this work for the nitrogen trifluoride ${ }^{14} \mathrm{NF}_{3}$ are given in Table 3.

The experimental centre of the $2 v_{2}$ band was estimated from the ${ }^{Q} Q$ branches edge at $1292.26 \mathrm{~cm}^{-1}$. But a precise value can be obtained combining the values of the experimental centres of $v_{2}$ and $2 v_{2}-v_{2}$ (Table 2). From the relationships:

$$
\begin{gathered}
\left(2 v_{2}\right)^{0}=2\left(v_{2}\right)^{0}+2 x_{22}, \\
\left(2 v_{2}-v_{2}\right)^{0}=\left(v_{2}\right)^{0}+2 x_{22},
\end{gathered}
$$


TABLE 3: Anharmonicity constants of ${ }^{14} \mathrm{NF}_{3}\left(\right.$ in $\left.\mathrm{cm}^{-1}\right)$.

\begin{tabular}{lccc}
\hline Anharmonicity constant & Experimental value, this work & Ab initio TZ2Pf [18] & Experimental value [20, 21] \\
\hline$x_{11}$ & - & -3.15 & -2.9 \\
$x_{12}$ & - & -3.56 & -4 \\
$x_{13}$ & $-7.965051(26)$ & -8.14 & -9.9 \\
$x_{14}$ & $-2.383248(49)$ & -2.08 & -1.5 \\
$x_{22}$ & $-1.006059(11)$ & -0.88 & - \\
$x_{23}$ & $-8.53^{\mathrm{a}}$ & -6.46 & -7.6 \\
$x_{24}$ & $-2.280208(11)$ & -1.98 & -2.5 \\
$x_{33}$ & $-4.15278(18)$ & -4.19 & - \\
$x_{34}$ & - & -5.12 & - \\
$x_{44}$ & $-0.841743(22)$ & -0.79 & -0.820 \\
$g_{33}$ & $1.82344(18)$ & 1.96 & - \\
$g_{34}$ & - & -0.10 & - \\
$g_{44}$ & $0.730149(22)$ & 0.72 & -1 \\
$x_{34}+g_{34}$ & - & - & 0.729 \\
\hline
\end{tabular}

Numbers in parentheses are one standard deviation in units of the last digit quoted.

${ }^{a}$ Value deduced from the $\left(v_{2}+v_{3}\right)^{0}$ given in Table 2.

TABLE 4: Harmonic wavenumber constants of ${ }^{14} \mathrm{NF}_{3}\left(\right.$ in $\left.\mathrm{cm}^{-1}\right)$.

\begin{tabular}{lccc}
\hline Harmonic wavenumber & Experimental value, this work & Ab initio unscaled value, [22] & $A b$ initio value TZ2Pf, [18] \\
\hline$\omega_{1}{ }^{0}$ & - & 1042.8 & 1053.8 \\
$\omega_{2}{ }^{0}$ & $648.140120(18)$ & 659.3 & 660.7 \\
$\omega_{3}{ }^{0}$ & $909.84264(43)$ & 905.0 & 935.7 \\
$\omega_{4}{ }^{0}$ & $493.534359(52)$ & 500.6 & 502.8 \\
\hline
\end{tabular}

Numbers in parentheses represent one standard deviation in units of the last digit quoted.

we can indeed deduce that $\left(2 v_{2}\right)^{0}=1292.25004(21) \mathrm{cm}^{-1}$ and $x_{22}=-1.006059$ (11).

The values obtained for $x_{22}, x_{44}$, and $g_{44}$ are small suggesting that the corresponding levels $v_{2}=2$ and $v_{4}=2$ are not significantly affected by the anharmonic perturbations, whereas the vibrational dependence is extremely important for the $v_{1}=v_{3}=1$ and $v_{2}=v_{3}=1$ levels.

It is worth noting that our values of the anharmonicity constants of ${ }^{14} \mathrm{NF}_{3}$ are in good agreement with previous medium experimental studies (column 4 of Table 3), but significantly more accurate by at least three orders of magnitude.

One can notice the fair agreement between our values and those obtained by ab initio methods [18] employing the TZ2Pf basis.

We can also extract accurate values of the harmonic wavenumbers of the oblate symmetric top molecule ${ }^{14} \mathrm{NF}_{3}$. The results are gathered in Table 4 .

The agreement between our values and those determined by the theoretical $a b$ initio calculations is significantly worse. We think that it is necessary to give much more credibility to our $\omega_{i}^{0}$ values which were deduced from experimental constants.

\section{Conclusion}

Using the recent accurate results obtained for the bands below $2000 \mathrm{~cm}^{-1}$, of the symmetric top molecule ${ }^{14} \mathrm{NF}_{3}$, several rovibrational interaction and anharmonicity constants have been deduced.

Our results contribute incontestably to the experimental knowledge of the molecular potential of this molecule which helps to test and improve theoretical models.

\section{References}

[1] H. Reichardt, A. Frenzel, and K. Schober, "Environmentally friendly wafer production: $\mathrm{NF}_{3}$ remote microwave plasma for chamber cleaning," Microelectronic Engineering, vol. 56, no. 1-2, pp. 73-76, 2001.

[2] V. Fthenakis, D. O. Clark, M. Moalem et al., "Life-cycle nitrogen trifluoride emissions from photovoltaics," Environmental Science and Technology, vol. 44, no. 22, pp. 8750-8757, 2010.

[3] M. J. Prather and J. Hsu, "Correction to ' $\mathrm{NF}_{3}$, the greenhouse gas missing from Kyoto,' Geophysical Research Letters, vol. 37, no. $11,2010$.

[4] T. Wen-Tien, "Environmental and health risk analysis of nitrogen trifluoride $\left(\mathrm{NF}_{3}\right)$, a toxic and potent greenhouse gas," Journal of Hazardous Materials, vol. 159, no. 2, pp. 257-263, 2008.

[5] M. J. Prather and J. Hsu, " $\mathrm{NF}_{3}$, the greenhouse gas missing from Kyoto," Geophysical Research Letters, vol. 35, no. 12, 2008.

[6] R. F. Weiss, J. Mühler, P. K. Salameh, and C. M. Harth, "Nitrogen trifluoride in the global atmosphere," Geophysical Research Letters, vol. 35, no. 20, 2008. 
[7] J. I. Robson, L. K. Gohar, M. D. Hurley, K. P. Shine, and T. Wallington, "Revised IR spectrum, radiative efficiency and global warming potential of nitrogen trifluoride," Geophysical Research Letters, vol. 33, no. 10, 2006.

[8] H. Najib, S. Hmimou, and H. Msahal, "High-resolution infrared spectroscopy of the $v_{1}+v_{4}$ band of ${ }^{14} \mathrm{NF}_{3}$ : reductions of the rovibrational hamiltonian," European Journal of Chemistry, vol. 9, no. 1, pp. 253-259, 2012.

[9] S. Hmimou, H. Msahal, and H. Najib, "First high-resolution FTIR study of the $v_{1}=v_{4}=1$ rovibrational state of ${ }^{14} \mathrm{NF}_{3}$ near $1523 \mathrm{~cm}^{-1}$," Molecular Physics, vol. 108, no. 6, pp. 787-794, 2010.

[10] N. B. Sari-Zizi and H. Najib, "High-resolution infrared study of the $2 v_{3}\left(A_{1}, E\right)$ and $v_{1}+v_{3}(E)$ bands of ${ }^{14} \mathrm{NF}_{3}$," Journal of Molecular Spectroscopy, vol. 240, no. 2, pp. 210-226, 2006.

[11] N. B. Sari-Zizi, H. Najib, J. Demaison, B. Bakri, J. M. Colmont, and H. Bürger, "High-resolution FTIR and MMW study of the $v_{4}=2\left(\mathrm{~A}_{1}, \mathrm{E}\right)$ excited state of ${ }^{14} \mathrm{NF}_{3}$ near $985 \mathrm{~cm}^{-1}$ : the axial ground state rotational constants derived by the "loopmethod'"' Journal of Molecular Spectroscopy, vol. 228, no. 2, pp. 511-527, 2004.

[12] H. Najib, N. B. Sari-Zizi, J. Demaison, B. Bakri, J. M. Colmont, and E. B. MKadmi, "High-resolution infrared and millimeterwave spectra of the $v_{3}=1$ vibrational state of ${ }^{14} \mathrm{NF}_{3}$ at $907 \mathrm{~cm}^{-1}$," Journal of Molecular Spectroscopy, vol. 220, no. 2, pp. 214-222, 2003.

[13] K. Akkad, N. B. Sari-Zizi, B. Bakri, J. Demaison, H. Bürger, and E. B. Mkadmi, "Fourier transform infrared and millimeter-wave study of the $v_{2}=1 v_{2}=1,2$ and the $v_{2}=v_{4}=1 v_{2}=v_{4}=1$ rovibrational states of ${ }^{14} \mathrm{NF}_{3}$," Journal of Molecular Spectroscopy, vol. 218, no. 1, pp. 36-47, 2003.

[14] N. Boulaftali, N. B. Sari-Zizi, U. Wötzel et al., "The $4_{4}=1$ state of ${ }^{14} \mathrm{NF}_{3}$ at $493 \mathrm{~cm}^{-1}$ studied by high-resolution FTIR, centimeterwave, and millimeter-wave spectroscopy," Journal of Molecular Spectroscopy, vol. 212, no. 1, pp. 41-52, 2002.

[15] H. Najib, "Vibrational constants of the symmetric top molecule $\mathrm{PF}_{3}$," Molecular Physics, vol. 106, no. 9-10, pp. 1199-1204, 2008.

[16] Z. Kisiel, M. Yu-Tretyakov, J. Demaison, L. Margulès, and P. Pracna, "High-resolution FTIR and millimeter-wave study of D3SiF: the ground, $v_{3}=1$, and $v_{6}=1$ and 2 states," Journal of Molecular Spectroscopy, vol. 197, no. 1, pp. 85-99, 1999.

[17] W. Höhe, U. Häring, W. A. Kreiner, H. Essig, and A. Ruoff, "Analysis of the IR spectrum of $\mathrm{NF}_{3}$ combining FT and laser saturation spectroscopy," Canadian Journal of Physics, vol. 72, pp. 1051-1059, 1994.

[18] J. Breidung, L. Constantin, J. Demaison, L. Margulès, and W. Thiel, "Ground state rotational spectrum, $\mathrm{K}=3$ splittings, $\mathrm{ab}$ initio anharmonic force field and equilibrium structure of trifluoroamine," Molecular Physics, vol. 101, no. 8, pp. 1113-1122, 2003.

[19] G. Cazzoli and C. Puzzarini, "Ground state rotational spectrum of nitrogen trifluoride: the $\mathrm{K}=3$ splittings of ${ }^{14} \mathrm{NF}_{3}$ and ${ }^{15} \mathrm{NF}_{3}$," Journal of Molecular Spectroscopy, vol. 239, no. 1, pp. 59-63, 2006.

[20] R. J. L. Popplewell, F. N. Masri, and H. W. Thompson, "Infrared spectrum and molecular constants of nitrogen trifluoride," Spectrochimica Acta A, vol. 23, no. 11, pp. 2797-2807, 1967.

[21] R. Reichman and J. Schatz, "l-type resonance in an overtone band. The $2 \mathrm{v}_{4}$ spectrum of nitrogen trifluoride," Journal of Molecular Spectroscopy, vol. 48, no. 2, pp. 277-282, 1973.
[22] R. Tarroni, P. Palmieri, M. L. Senent, and A. Willets, "Ab initio calculation of the anharmonic force and dipole fields of nitrogen trifluoride," Chemical Physics Letters, vol. 257, no. 1, pp. 23-30, 1996. 

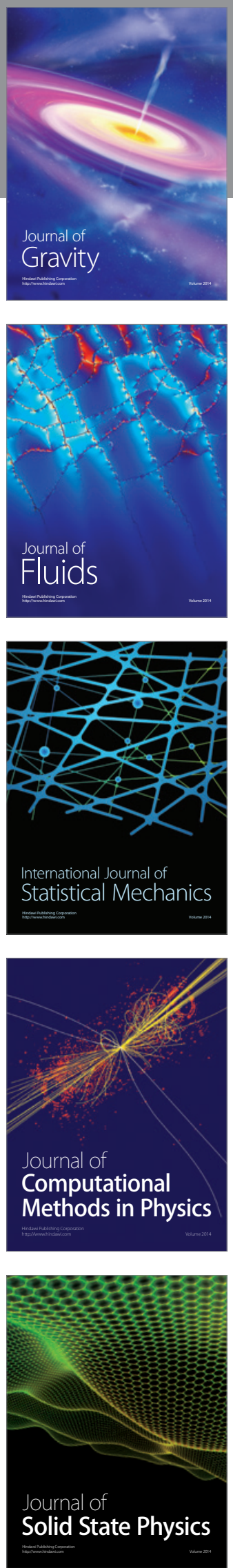

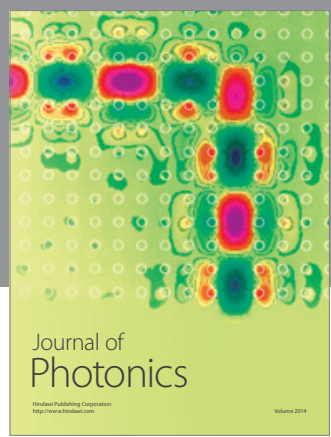

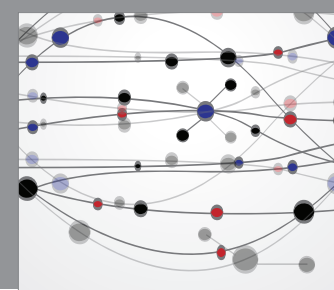

The Scientific World Journal

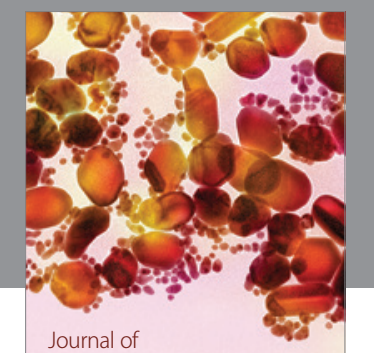

Soft Matter
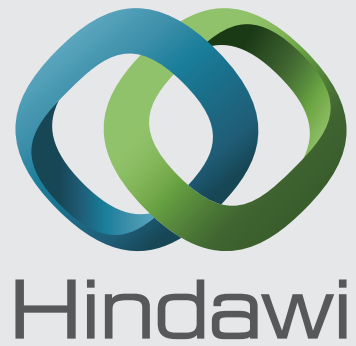

Submit your manuscripts at

http://www.hindawi.com
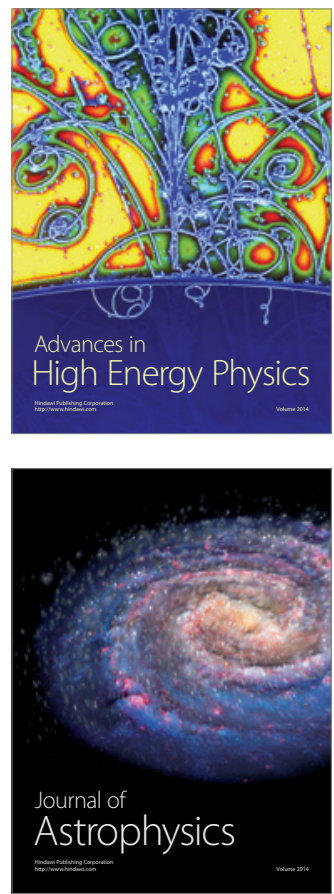
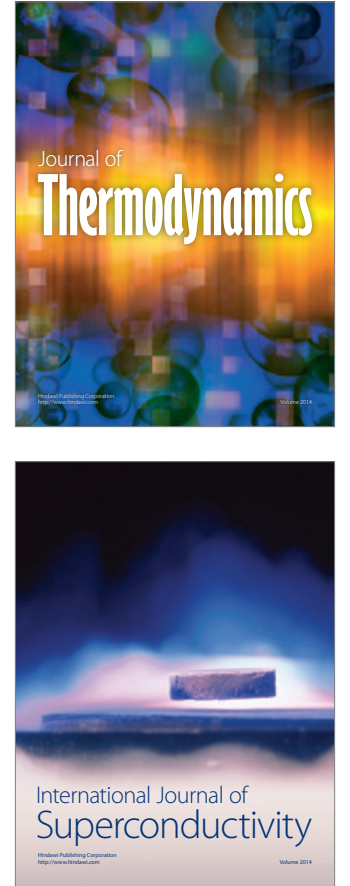
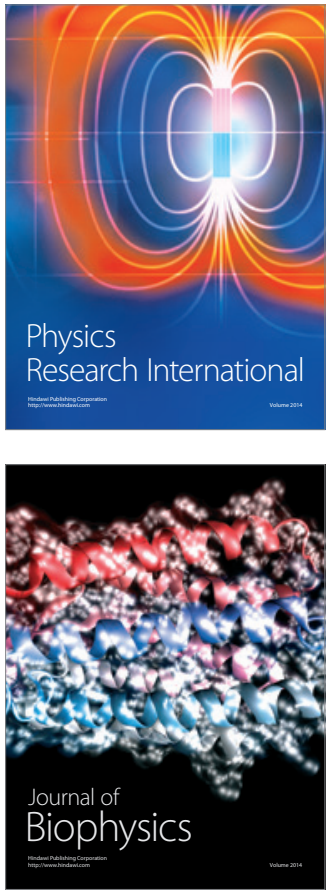
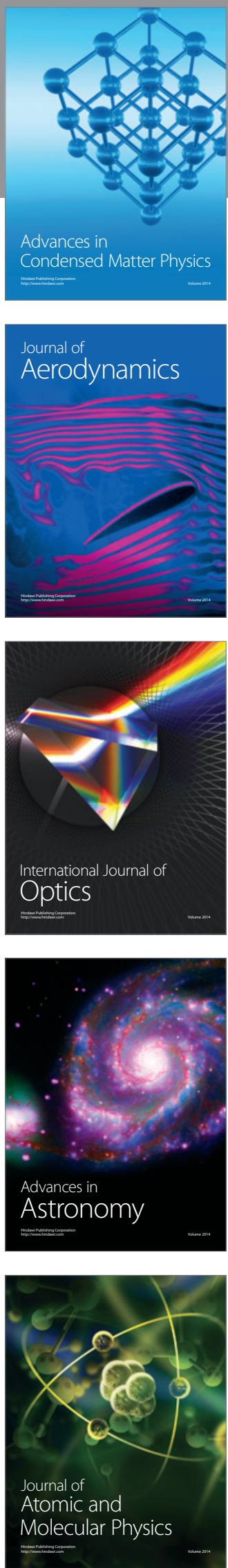